\title{
Decays of polarized top quarks to lepton, neutrino, and jets at NLO QCD
}

\author{
W. Bernreuther ${ }^{1, a}$, P. González ${ }^{2}$, C. Mellein ${ }^{1, b}$ \\ ${ }^{1}$ Institut für Theoretische Physik, RWTH Aachen University, 52056 Aachen, Germany \\ 2 Present address: Department of Radiation Oncology, Netherlands Cancer Institute, 1066 CX Amsterdam, The Netherlands
}

Received: 24 January 2014 / Accepted: 6 March 2014 / Published online: 19 March 2014

(C) The Author(s) 2014. This article is published with open access at Springerlink.com

\begin{abstract}
We compute the differential and total rate of the semileptonic decay of polarized top quarks $t \rightarrow \ell \nu_{\ell}+b$ jet + jet at next-to-leading order (NLO) in the QCD coupling with an off-shell intermediate $W$ boson. We present several normalized distributions, in particular those that reflect the $t$-spin analyzing powers of the lepton, the b-jet and the $W^{+}$boson at LO and NLO QCD.
\end{abstract}

\section{Introduction}

The top quark, the heaviest known fundamental particle, is set apart from the lighter quarks by the fact that it is so shortlived that it does not hadronize. The top quark decays almost exclusively into a $b$ quark and a $W$ boson; other decay modes have so far not been observed.

Top-quark production and decay has been explored quite in detail at the Tevatron and especially at the Large Hadron Collider (LHC). So far almost all experimental results agree well with corresponding Standard Model (SM) predictions. (For recent overviews, see [1-4].) On the theoretical side, significant recent progress ${ }^{1}$ includes the computation of the hadronic $t \bar{t}$ production cross section at next-to-next-toleading order (NNLO) in the QCD coupling $\alpha_{s}[5,6]$ and the calculation of the differential decay rate of $t \rightarrow b \ell v_{\ell}$ at NNLO in perturbative QCD $[7,8]$.

Over the years, top-quark decay has been analyzed in detail within the SM. As to the total decay width $\Gamma_{t}$, the order $\alpha_{s}$ QCD corrections $[9,10]$, the order $\alpha$ electroweak corrections [11,12], and the order $\alpha_{s}^{2}$ QCD corrections [13,14] were calculated quite some time ago. The fractions of top-quark decay into $W^{+}$with helicity $\lambda_{W}=0, \pm 1$ are also known to NNLO QCD [15], including the order $\alpha$ electroweak cor-

\footnotetext{
a e-mail: breuther@physik.rwth-aachen.de

b e-mail: mellein@physik.rwth-aachen.de

${ }^{1}$ Other recent results include $[42,43]$.
}

rections [16]. Differential distributions of semileptonic and non-leptonic decays of (un)polarized top quarks were determined to NLO in the gauge couplings [17-25], and $b$-quark fragmentation was analyzed in [26-30].

In this paper we compute the differential and total rate of polarized top quarks decaying into $\ell v_{\ell}+b$ jet + jet at NLO in the QCD coupling. The differential rate is of interest as a building block for predictions of top-quark production and decay at NLO QCD, for instance for $t \bar{t}+$ jet production [3133], for single top-quark + jet production at the LHC, or for $t \bar{t}+$ jet production at a future $e^{+} e^{-}$linear collider. In fact, this decay mode was already computed to NLO QCD by [33]. The results of this paper as regards $t \bar{t}+$ jet production at hadron colliders include also NLO jet radiation in top-quark decay. Distributions for this decay mode were not given separately in [33]. Therefore, we believe that it is useful to present, for possible applications to other processes, a separate detailed analysis of this top-quark decay mode.

The paper is organized as follows. In Sect. 2 we describe our computational setup. In Sect. 3 we present our results for the decay rate and for a number of distributions for (un)polarized top-quark decays. Section 4 contains a short summary. In the appendix we list the subtraction terms, for the Catani-Seymour subtraction formalism $[34,35]$ with extensions to the case of a colored massive initial state $[33,36,37]$, which we use to handle the soft and collinear divergences that appear in the real radiation and NLO virtual correction matrix elements.

\section{Setup of the computation}

We consider the decay of polarized top quarks to leptons, a $b$-jet, and an additional jet,

$t \rightarrow W^{*+}+b$ jet + jet $\rightarrow \ell^{+} \nu_{\ell}+b$ jet + jet,

at NLO QCD, for an off-shell intermediate $W$ boson. The quarks and leptons in the final state are taken to be massless. 
At NLO QCD, i.e., at order $\alpha_{s}^{2}$, the differential decay rate of (1) is determined by the amplitudes of the following parton processes:

$t \rightarrow \ell^{+} v_{\ell}+b+g$

and

$t \rightarrow \ell^{+} v_{\ell}+b+g g, \quad t \rightarrow \ell^{+} v_{\ell}+b+q \bar{q}, \quad q=u, d, s, c, b$.

In the case of additional $b \bar{b}$ production in the real radiation process (3), we take into account only configurations where a $b \bar{b}$ pair is unresolved by the jet algorithm, i.e., we consider in (1) final states where the additional jet has zero $b$-flavor. To order $\alpha_{s}^{2}$, the matrix element of (2) is the sum of the Born term $\left|\mathcal{M}_{B}\right|^{2}$ and the interference $\delta \mathcal{M}_{V}$ of the Born and the 1-loop amplitude. The calculation of $\delta \mathcal{M}_{V}$, using dimensional regularization, is standard. We expressed $\delta \mathcal{M}_{V}$, using Passarino-Veltman reduction [38], in terms of scalar one-loop integrals with up to four external legs. The scalar integrals that appear in $\delta \mathcal{M}_{V}$ are known analytically in $d$ space-time dimensions (cf., for instance, [39] and references therein). In particular, we extracted the ultraviolet (UV) and infrared (IR) poles in $\epsilon=(4-d) / 2$ that appear in several of these scalar integrals analytically. The processes (3) are described by tree-level matrix elements.

As to renormalization, the top-quark mass is defined in the on-shell scheme while the QCD coupling $\alpha_{s}$ is defined in the $\overline{\mathrm{MS}}$ scheme.

The soft and collinear divergences that appear in the phasespace integrals of the tree-level matrix elements of (3) and in $\delta \mathcal{M}_{V}$ are handled with the dipole subtraction method [34,35] and extensions that apply to the decay of a massive quark $[33,36,37]$. Details are given in the appendix.

We define jets by the Durham algorithm [40], i.e., we use the jet metric

$Y_{i j}=2 \frac{\min \left(E_{i}^{2}, E_{j}^{2}\right)}{m_{t}^{2}}\left(1-\cos \theta_{i j}\right)$,

where $E_{i}, E_{j}$ are the energies of partons $i, j$ in the final state, $\theta_{i j}$ is the angle between them, and $m_{t}$ denotes the mass of the top quark. We work in the rest frame of the $t$ quark. The jet resolution parameter is denoted by $Y$. An unresolved pair of final-state partons $i, j$ is recombined by adding the fourmomenta, $k_{(i j)}=k_{i}+k_{j}$. The decay (2) contributes to (1) events with $Y_{b g}>Y$. The real radiation processes (3) contribute to (1) events with one unresolved pair of partons $i, j$, i.e., with $Y_{i j}<Y$. The jet distance between the recombined pseudoparticle $(i j)$ and the remaining parton $n$ must satisfy $Y_{n(i j)}>Y$.

\section{Results}

As already mentioned, we work in the top-quark rest frame. If we denote the top-spin vector in this frame by $\mathbf{s}_{t}$ (where $\mathbf{s}_{t}^{2}=1$ ), differential distributions for the decay (1) of a $100 \%$ polarized ensemble of top quarks are of the form

$\frac{\mathrm{d} \Gamma}{\mathrm{d} O}=A+\mathbf{B} \cdot \mathbf{s}_{t}$

where $O$ denotes some observable. In the fully differential case, the functions $A$ and $\mathbf{B}$ (which transform as scalar and vector, respectively, under spatial rotations) depend on the independent kinematical variables of (1), and the vector $\mathbf{B}$ may be represented as a linear combination of terms proportional to the directions of the charged lepton and of the two jets in the final state.

Rotational invariance implies that a number of distributions hold both for polarized and unpolarized top quarks. This includes the distributions that will be presented in Sect. 3.1.

In Sect. 3.2 we consider distributions that are relevant for the decay of polarized top quarks, namely those that reflect the top-spin analyzing power of the charged lepton, the b-jet, and the $W$ boson.

For the numerical results given below, we use $m_{t}=$ $173.5 \mathrm{GeV}, m_{W}=80.39 \mathrm{GeV}$ and $\Gamma_{W}=2.08 \mathrm{GeV}$. The QCD coupling for 5-flavor QCD is taken to be $\alpha_{s}\left(m_{Z}\right)=$ 0.118 . Its evolution to $\mu=m_{t}$ and conversion to the 6-flavor $\overline{\mathrm{MS}}$ coupling results in $\alpha_{s}\left(m_{t}\right)=0.108$. Moreover, we use $\alpha\left(m_{t}\right)=7.9 \times 10^{-3}$ and $\sin ^{2} \theta_{W}=0.231$ which yields the weak coupling $g_{W}^{2}=0.429$. The normalized distributions given below do not depend on $g_{W}^{2}$ because we work to lowest order in $g_{W}^{2}$.

\subsection{Distributions for (un)polarized top-quark decay}

First, we compute the decay rate of (1) as a function of the jet resolution parameter $Y$. In Fig. 1 the ratio of $\Gamma_{t \rightarrow b} \bar{l}_{l}+$ jet and the leading order rate $\Gamma_{t \rightarrow b \bar{l} \nu_{l}}^{L O}=0.162 \mathrm{GeV}$ is shown at LO and NLO QCD, for a renormalization scale $\mu=m_{t}$. It is clear that this ratio increases for decreasing $Y$. In the lower

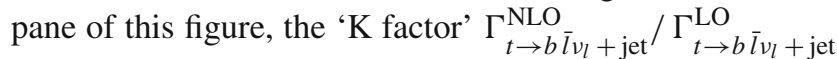
is displayed. One sees that in a large range of $Y$, the QCD corrections are positive and at most of order $8 \%$, while for $Y$ below $\sim 2.5 \times 10^{-3}$ they become negative.

In the remainder of this section we compute normalized decay distributions, both at LO and NLO QCD for two values of the jet resolution parameter, $Y=0.01$ and $Y=0.001$. The NLO decay distributions, which are normalized to the NLO decay rate $\Gamma_{t \rightarrow b}^{\mathrm{NLO}} \bar{l}_{\nu_{l}+\text { jet }}$, are expanded in powers of $\alpha_{s}$. Taking out a factor of $\alpha_{s}$ both from the LO and NLO (differential) rate, we have 


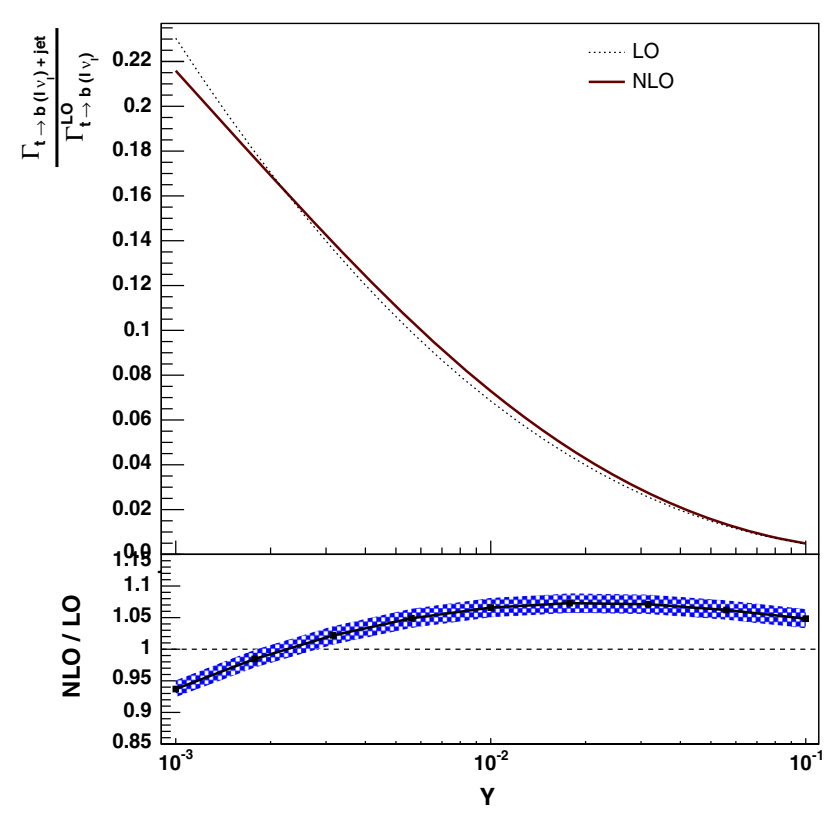

Fig. 1 Upper pane decay rate $\Gamma_{t \rightarrow b} \bar{l}_{\nu_{l}}+$ jet (LO and NLO) normalized to $\Gamma_{t \rightarrow b \bar{l}_{v_{l}}}$ (LO) as a function of the jet resolution parameter $Y$ for $\mu=m_{t}$. Lower pane ratio of $\Gamma_{t \rightarrow b \bar{l}_{v_{l}}+\text { jet }}^{\mathrm{NLO}} / \Gamma_{t \rightarrow b \bar{l} v_{l}+\text { jet }}^{\mathrm{LO}}$ as a function of $Y$. The solid line corresponds to $\mu=m_{t}$, the shaded band results from scale variations between $m_{t} / 2$ and $2 m_{t}$

$$
\begin{aligned}
\frac{\mathrm{d} \Gamma^{\mathrm{NLO}}}{\Gamma^{\mathrm{NLO}}} & =\frac{\mathrm{d} \Gamma_{0}+\alpha_{s} \mathrm{~d} \Gamma_{1}+\mathcal{O}\left(\alpha_{s}^{2}\right)}{\Gamma_{0}+\alpha_{s} \Gamma_{1}+\mathcal{O}\left(\alpha_{s}^{2}\right)} \\
& =\frac{\mathrm{d} \Gamma_{0}}{\Gamma_{0}}\left(1-\alpha_{s} \frac{\Gamma_{1}}{\Gamma_{0}}\right)+\alpha_{s} \frac{\mathrm{d} \Gamma_{1}}{\Gamma_{0}}+\mathcal{O}\left(\alpha_{s}^{2}\right) .
\end{aligned}
$$

In the following we rescale all dimensionful variables with $m_{t}$. That is, in the following, the energies $E_{W}, E_{l}, E_{b}$, and $E_{2}$ of the $W$ boson, the charged lepton, $b$-jet, and the second jet with zero $b$-flavor, respectively, and the $W$ and $\ell b$-jet invariant masses $M_{W}, M_{l b}$ denote dimensionless variables.

The invariant mass distribution and the energy distribution of the off-shell $W$ boson are displayed in Figs. 2 and 3 for $Y=0.01$ and $Y=0.001$, respectively. The QCD corrections to the invariant mass of the $W$ boson are very small. The distribution of the $W$ energy ${ }^{2} E_{W}=E_{l}+E_{v}$ may be compared with the case of the lowest-order on-shell decay $t \rightarrow b W$ where the (dimensionless) $W$ energy is fixed, $\bar{E}_{W}=\sqrt{m_{W}^{2}+\mathbf{k}_{W}^{2}} / m_{t}=0.61$. In the case of additional jet radiation and allowing the $W$ boson to be off-shell, one expects therefore that the maximum of the distribution of $E_{W}$ is below $\bar{E}_{W}$, but it approaches this value if the jet cut $Y$ is decreased. The distributions on the right sides of Figs. 2 and 3 show this behavior. The QCD corrections are small at and in the near vicinity of the maximum of the distribution, whereas

\footnotetext{
${ }^{2}$ Here, we tacitly assume that the neutrino energy and momentum can be reconstructed in an experiment, which is usually possible only with ambiguities.
}

they can become rather large if the $W$ boson is significantly off-shell.

The left sides of Figs. 4 and 5 show the distribution of the energy $E_{l}$ of the charged lepton. For decreasing jet cut the distribution moves toward the lepton-energy distribution of the inclusive semileptonic decay which, at tree level and for a massless $b$ quark, has its maximum at $E_{l}=0.25$.

The right sides of Figs. 4 and 5 display the distribution of the invariant mass $M_{l b}$ of the lepton and the $b \mathrm{jet}^{3}$. In the case of the LO decay $t \rightarrow b \ell v_{\ell}$ and an on-shell intermediate $W$ boson, $M_{l b}$ has a sharp upper bound, which, in terms of our dimensionless variables, is given by $M_{l b}^{\max }=\sqrt{1-m_{W}^{2} / m_{t}^{2}}$. In the case of (2), (3), where gluons or $q \bar{q}$ are radiated, the invariant mass $M_{l b}$ cannot exceed the LO kinematic boundary, as long as the $W$ boson is kept on-shell. The distance between the maximum of the $M_{l b}$ distribution and $M_{l b}^{\max }$ is expected to decrease with decreasing jet cut $Y$. An off-shell $W$ boson leads to a tail of the $M_{l b}$ distribution beyond $M_{l b}^{\max }$. All of these features arise in the results shown on the right sides of Figs. 4 and 5. In the vicinity of $M_{l b}^{\max }=0.89$ the QCD corrections are about $-10 \%$.

The distribution of the b-jet energy $E_{b}$ and of the energy $E_{2}$ of the second jet is displayed in Figs. 6 and 7. In the case of the LO decay $t \rightarrow b W$, the energy of the massless $b$ quark is fixed to be $\bar{E}_{b}=\left(1-m_{W}^{2} / m_{t}^{2}\right) / 2=0.39$. Radiation off the $t$ and $b$ leads to an upper bound on $E_{b}$ that is below $\bar{E}_{b}$ for $Y>0$. An off-shell $W$ boson can, however, lead to some events with $E_{b}$ above this value. The average energy $E_{2}$ of the second jet is smaller than that of the $b$ jet. These features are exhibited by the results shown in Figs. 6 and 7. Near the kinematic edges the QCD corrections can become $\sim 10 \%$.

Figures 8 and 9 show the distribution of $\cos \theta_{b l}$, where $\theta_{b l}$ is the angle between the directions of flight of the charged lepton and the b-jet in the $t$ rest frame, and of $\cos \theta_{2 l}$, where $\theta_{2 l}$ is the angle between $\ell^{+}$and the second jet. The distributions of $\cos \theta_{b l}$ are qualitatively similar to the corresponding distributions in the case of inclusive semileptonic top-decay; for most of the events the charged lepton and the $b$ jet are almost backto-back. As expected, the distribution of $\cos \theta_{2 l}$ is falling less steeply toward smaller angles $\theta_{2 l}$. The QCD corrections are markedly below $5 \%$ in most of the kinematic range.

The distribution of $\cos \theta_{W l}^{*}$, where $\theta_{W l}^{*}$ is the angle between the $W^{+}$direction in the $\mathrm{t}$ rest frame and the lepton direction in the $W^{+}$rest frame, is presented in the plots on the left side of Figs. 10 and 11. This distribution has been used ever since at the Tevatron and the LHC for measuring the $W$-boson helicity fractions in inclusive semileptonic topdecay. With $x=\cos \theta_{W l}^{*}$ the one-dimensional distribution has the well-known form

\footnotetext{
3 The distribution of $M_{l b}$ in inclusive hadronic $t \bar{t}$ production and decay was first analyzed at NLO QCD in [41] and was proposed as a tool to measure the top-quark mass. Cf. also [42].
} 

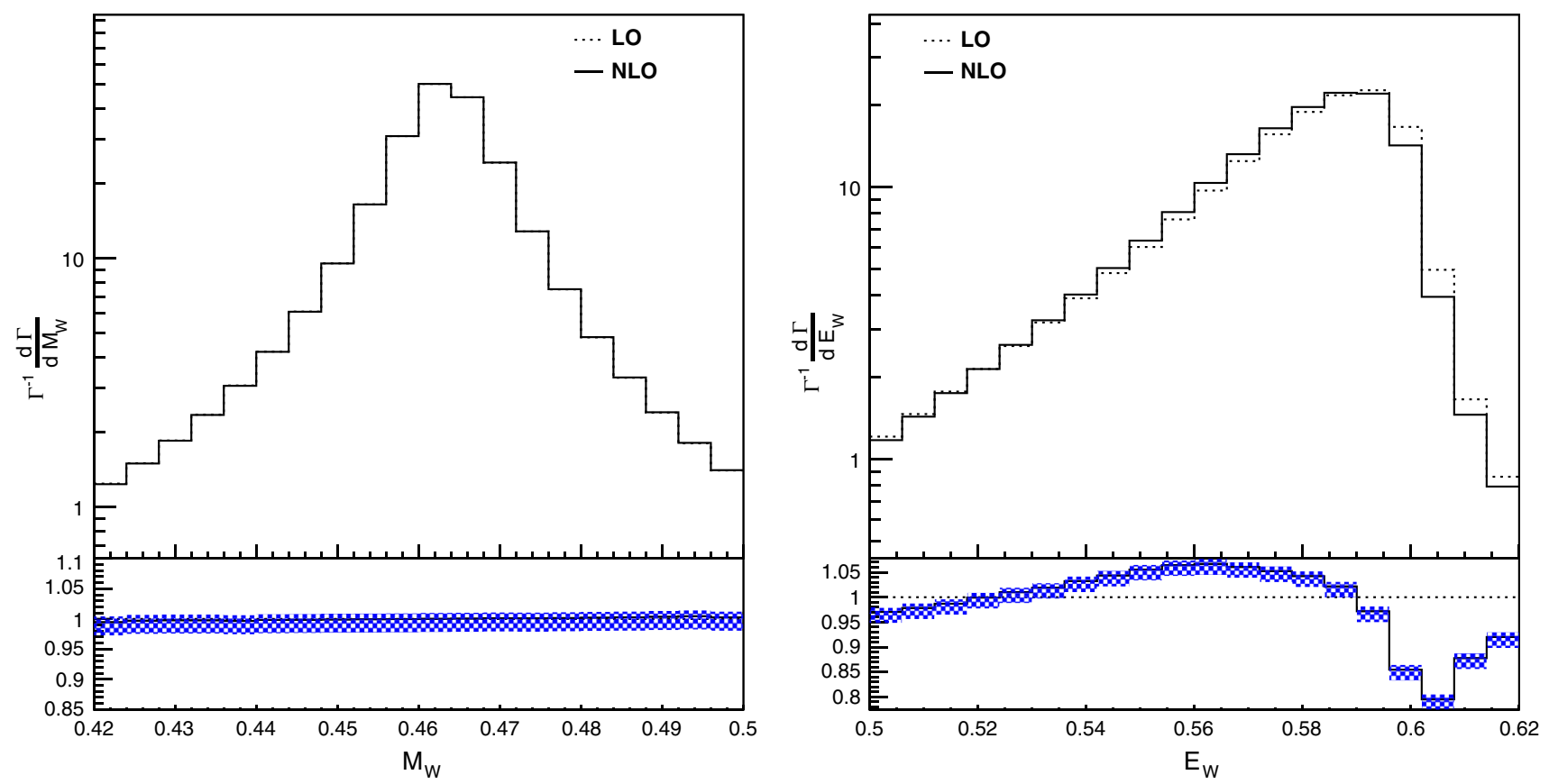

Fig. 2 Upper panes normalized distribution of the invariant mass $M_{W}$ of the $W$ boson $(l e f t)$ and of the $W$ energy $E_{W}($ right $)$ for $Y=0.01$ and $\mu=m_{t}$. Lower panes ratio of the NLO and corresponding LO distribution. The shaded band results from scale variations between $m_{t} / 2$ and $2 m_{t}$

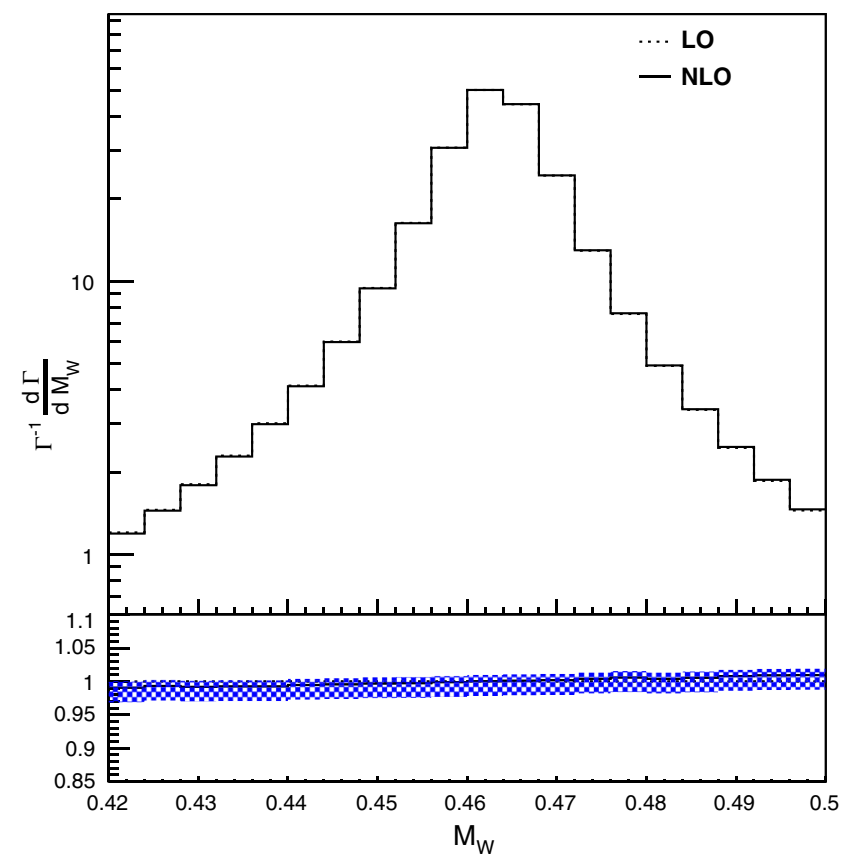

Fig. 3 Same as Fig. 2, but for a jet resolution parameter $Y=0.001$

$$
\begin{aligned}
\Gamma^{-1} \frac{\mathrm{d} \Gamma}{\mathrm{d} x}= & \frac{3}{4} F_{L}\left(1-x^{2}\right) \\
& +\frac{3}{8} F_{-}(1-x)^{2}+\frac{3}{8} F_{+}(1+x)^{2},
\end{aligned}
$$

with $F_{L}+F_{-}+F_{+}=1$. For events with an additional jet, one expects that for small jet cut $Y$ the corresponding distribution tends toward the inclusive one. Performing a fit to the $\cos \theta_{W l}^{*}$

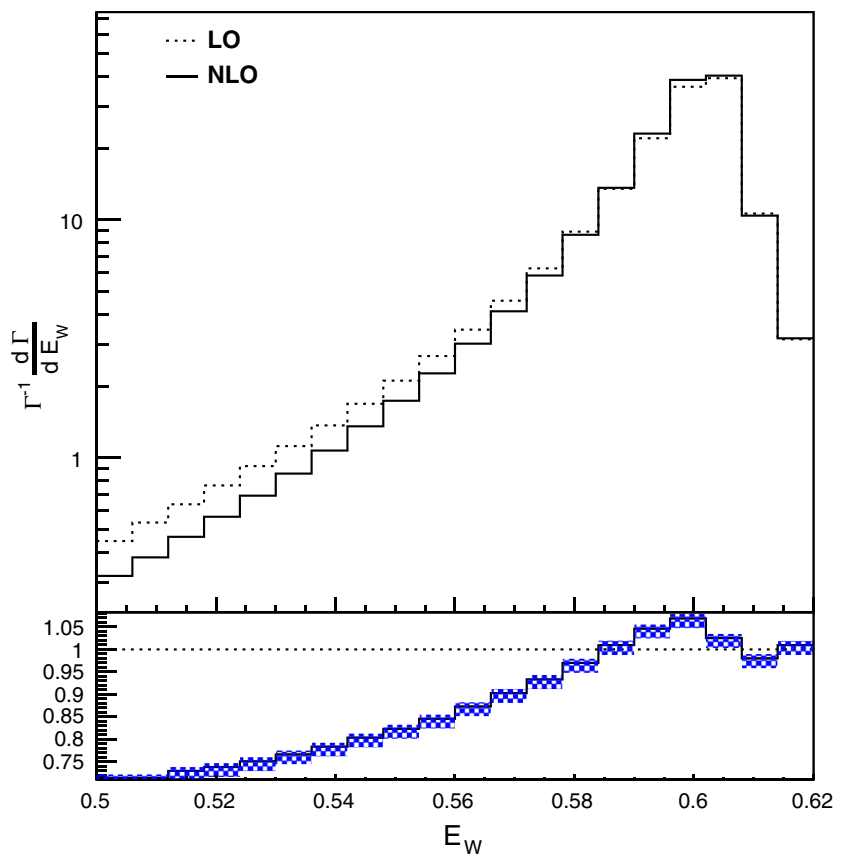

distributions of Figs. 10 and 11 (where we take into account that our NLO distributions are not exactly normalized to one, due to the expansion (6)), we obtain $F_{L}^{\mathrm{NLO}}=0.668$ and $F_{-}^{\mathrm{NLO}}=0.321$ for $Y=0.01$, and $F_{L}^{\mathrm{NLO}}=0.689$ and $F_{-}^{\mathrm{NLO}}=0.308$ for $Y=0.001$. The size of the QCD corrections is $\lesssim 1 \%$. For $Y=0.001$ the helicity fractions agree very well with the corresponding inclusive ones at NLO QCD (cf., 


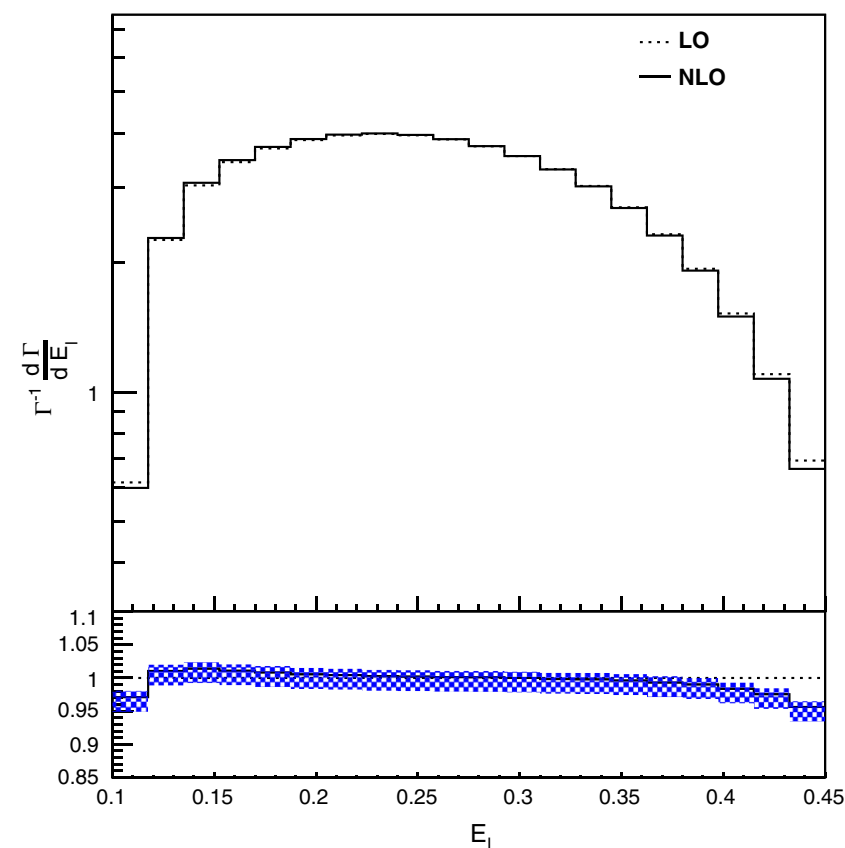

Fig. 4 Upper panes normalized distribution of the energy $E_{l}$ of the charged lepton (left) and of the invariant mass $M_{l b}$ of the $b$ jet and the charged lepton (right) for $Y=0.01$ and $\mu=m_{t}$. Lower panes ratio of

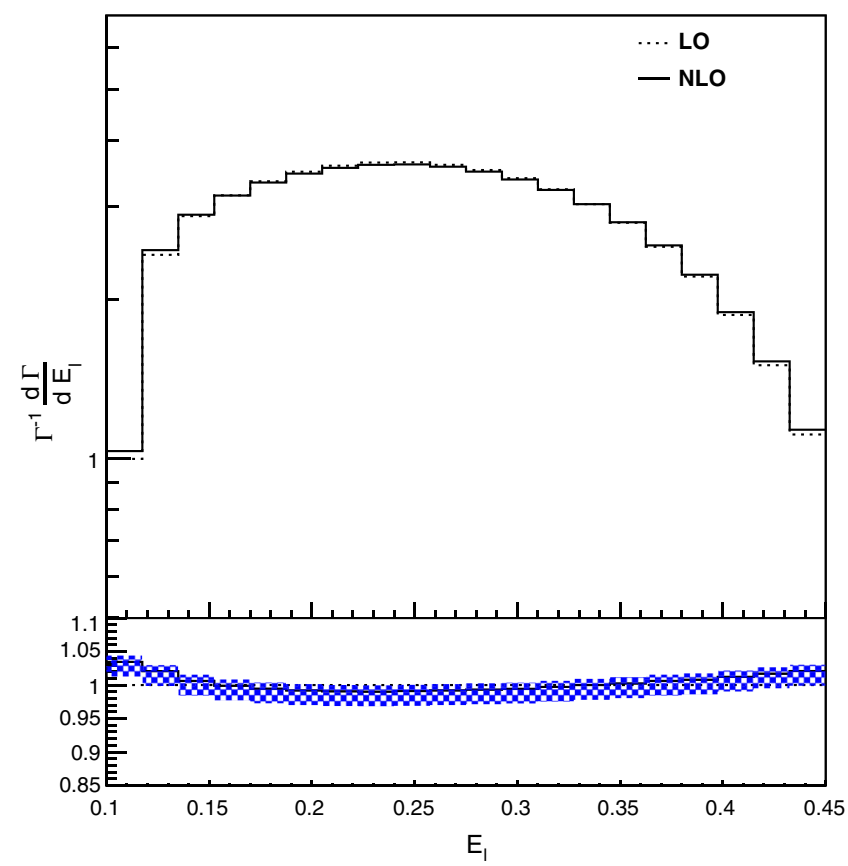

Fig. 5 Same as Fig. 4, but for $Y=0.001$

for instance, [15]) and are in agreement with recent results from ATLAS and CMS [44].

The plots on the right sides of Figs. 10 and 11 show the distribution of $\cos \theta_{W b}$, where $\theta_{W b}$ is the angle between the $W$ and the $\mathrm{b}$-jet directions in the $t$ rest frame. As in the inclusive case this distribution peaks when the $W$ boson and the $b$ jet are back-to-back.

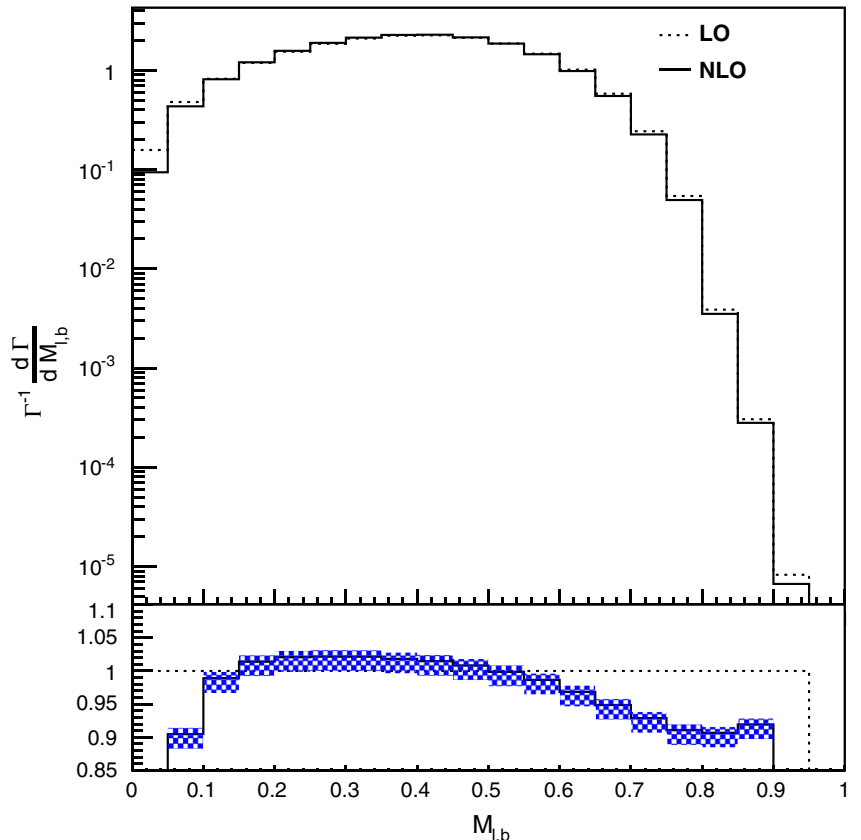

the NLO and corresponding LO distribution. The shaded band results from scale variations between $m_{t} / 2$ and $2 m_{t}$

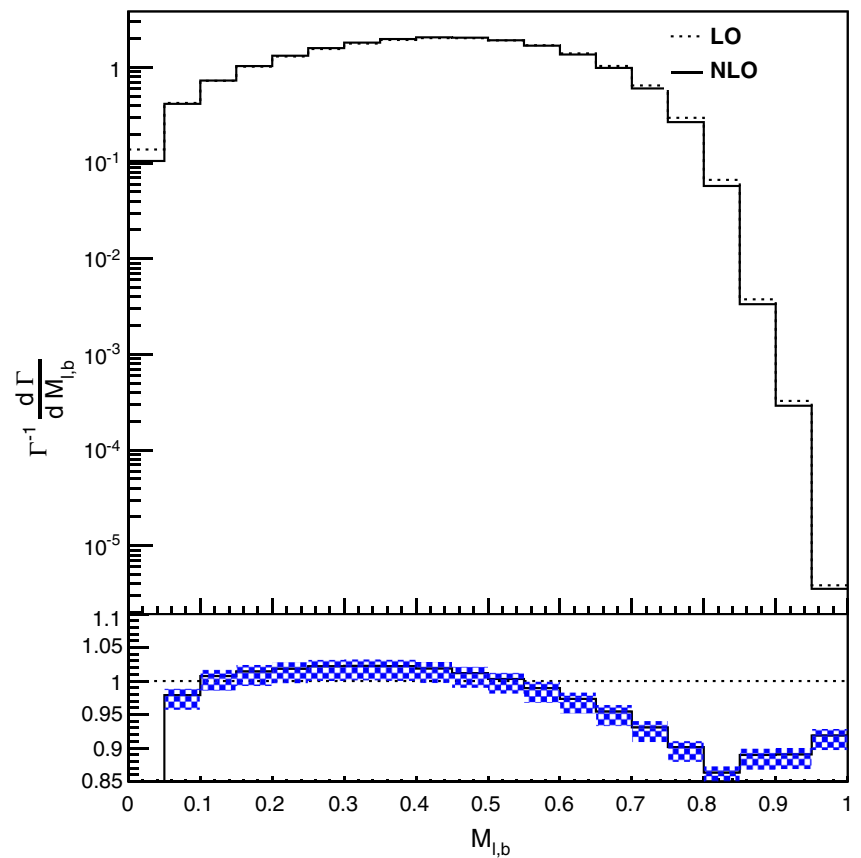

3.2 Top-spin analyzing power

Finally we consider, for the decay (1) of a $100 \%$ polarized top-quark ensemble, the angular correlation of the top-spin vector $\mathbf{s}_{t}$ and the direction of flight of a final-state particle or jet $f$ in the top rest frame, where $f=\ell^{+}, b$ jet, $W^{+}$. The corresponding normalized distribution has the a priori form 

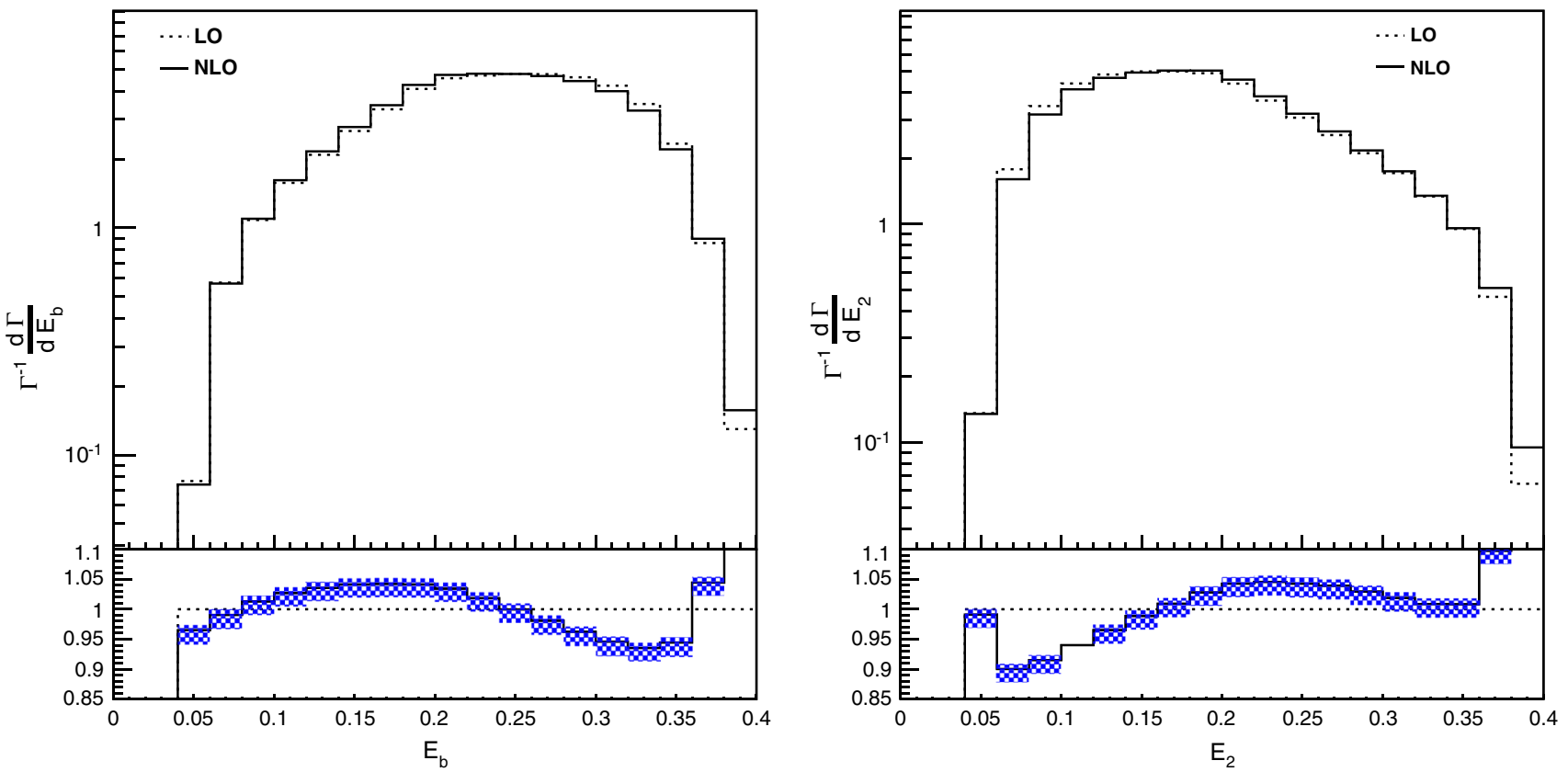

Fig. 6 Upper panes normalized distribution of b-jet energy $E_{b}$ (left) and of the energy $E_{2}$ of the second jet (right) for $Y=0.01$ and $\mu=m_{t}$. Lower panes ratio of the NLO and corresponding LO distribution. The shaded band results from scale variations between $m_{t} / 2$ and $2 m_{t}$

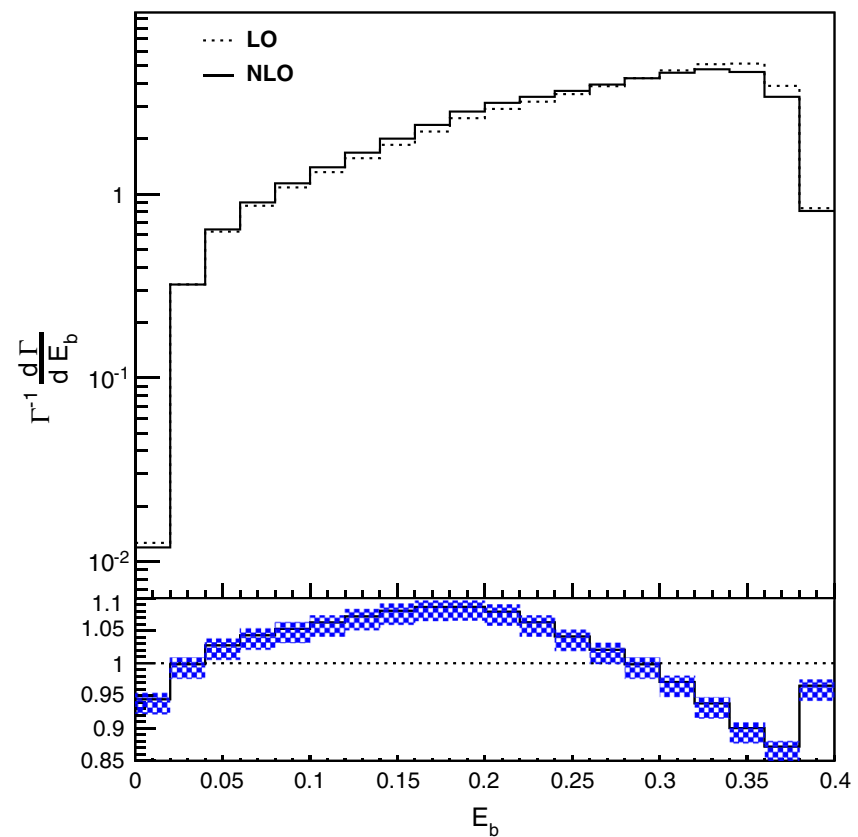

Fig. 7 Same as Fig. 6, but for $Y=0.001$

$$
\frac{1}{\Gamma} \frac{\mathrm{d} \Gamma}{\mathrm{d} \cos \theta_{f}}=\frac{1}{2}\left(1+\kappa_{f} \cos \theta_{f}\right),
$$

where $\theta_{f}=L\left(\mathbf{s}_{t}, \hat{\mathbf{k}}_{f}\right)$. The coefficient $\kappa_{f}$ is the top-spin analyzing power of $f$ and measures the degree of correlation. $\mathrm{CP}$ invariance implies ${ }^{4}$ that the corresponding angular

\footnotetext{
4 The effect of the non-zero Kobayashi-Maskawa phase, which would show up only if higher-order weak corrections are taken into account, is completely negligible in these decays.
}

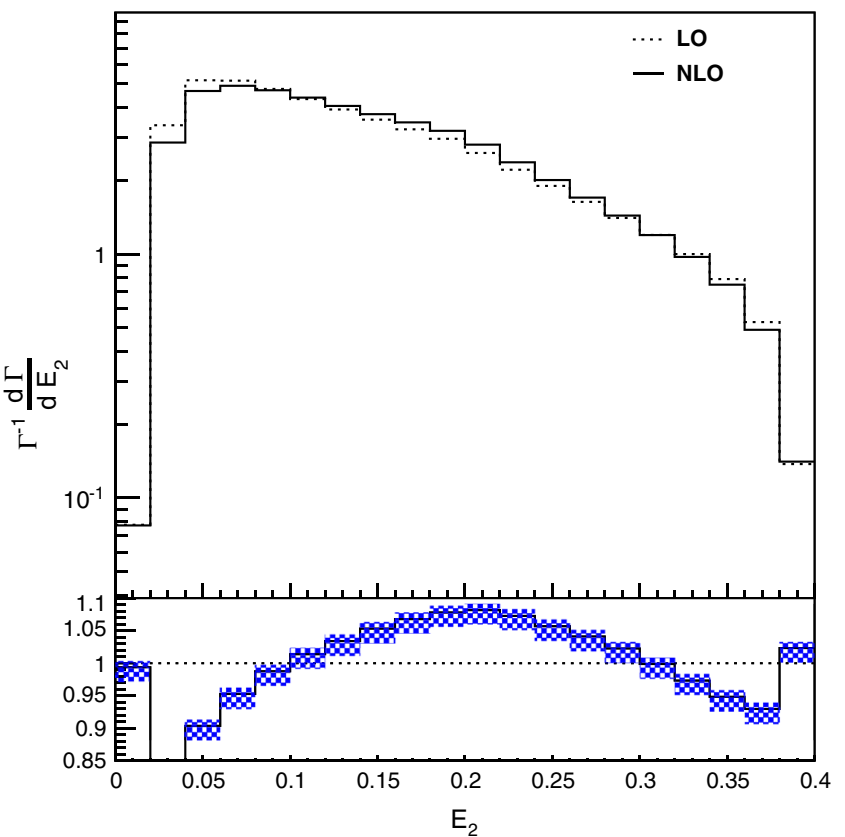

distributions for top antiquarks are given by

$\frac{1}{\bar{\Gamma}} \frac{\mathrm{d} \bar{\Gamma}}{\mathrm{d} \cos \theta_{\bar{f}}}=\frac{1}{2}\left(1-\kappa_{f} \cos \theta_{\bar{f}}\right)$.

The values of $\kappa_{f}$ can be extracted from the slope of the distributions (7) or from 


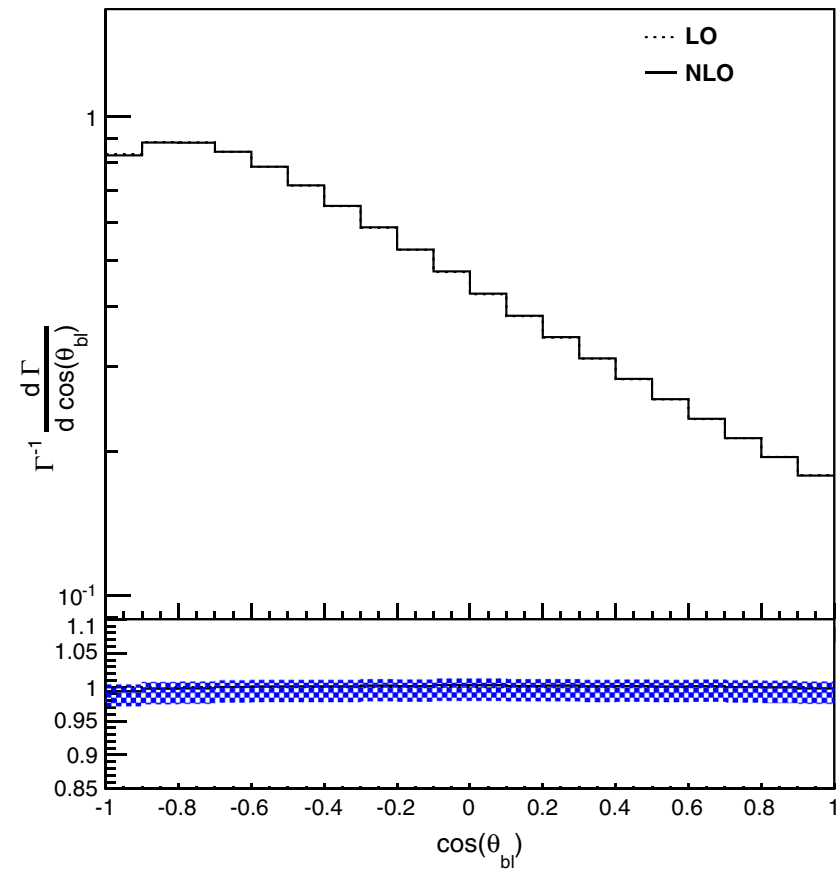

Fig. 8 Upper panes normalized distribution of $\cos \theta_{b l}$, where $\theta_{b l}$ is the angle between the directions of flight of the charged lepton and the b-jet in the $t$ rest frame (left), and of $\cos \theta_{2 l}$, where $\theta_{2 l}$ is the angle between $\ell^{+}$ and the second jet. The jet resolution parameter is chosen to be $Y=0.01$

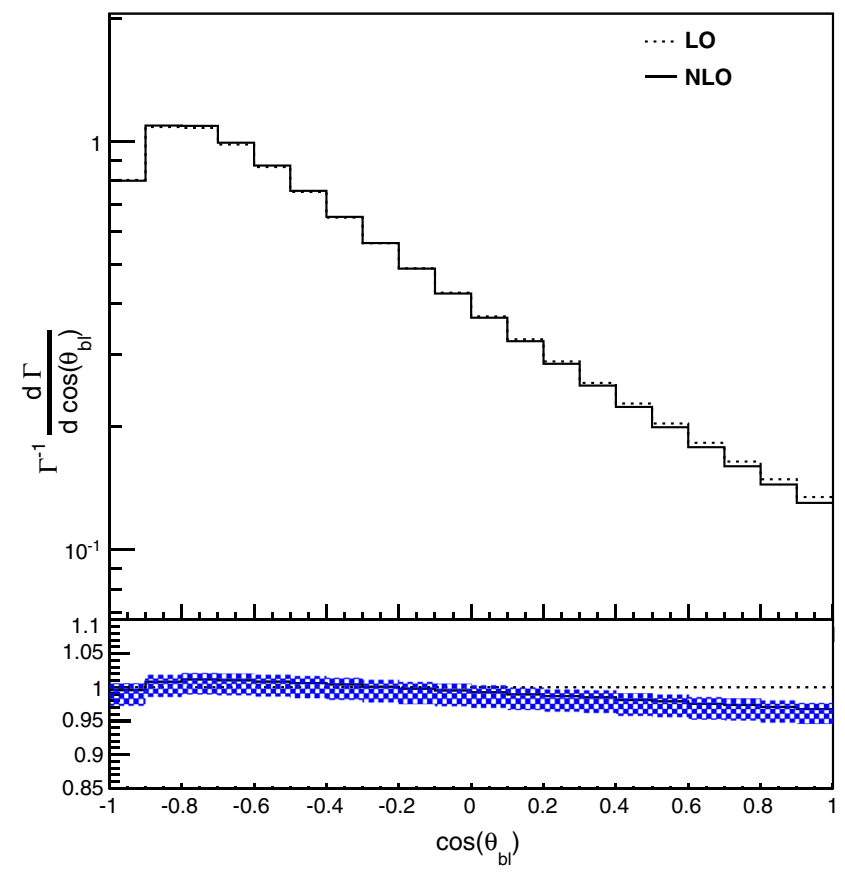

Fig. 9 Same as Fig. 8, but for $Y=0.001$

$\left\langle\cos \theta_{f}\right\rangle=\frac{1}{\Gamma} \int_{0}^{\pi} \mathrm{d} \cos \theta_{f}\left(\cos \theta_{f} \frac{\mathrm{d} \Gamma}{\mathrm{d} \cos \theta_{f}}\right)=\frac{\kappa_{f}}{3}$.

The results for $\kappa_{f}$ at LO and NLO QCD are listed in Table 1 for two values of the jet resolution parameter $Y$.

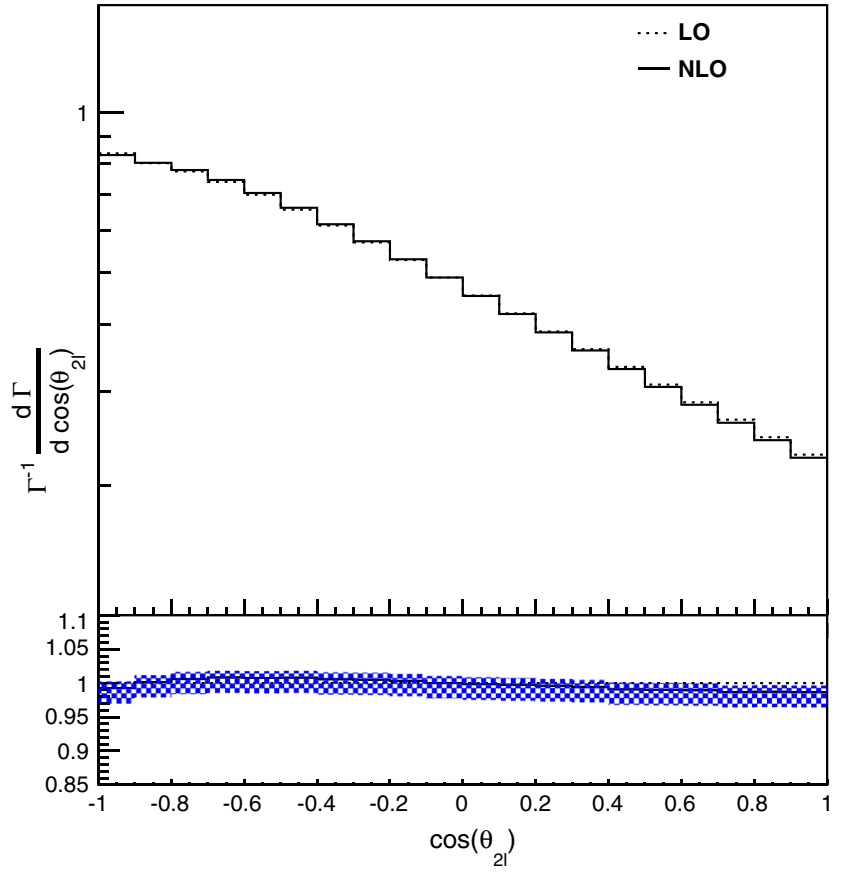

and $\mu=m_{t}$. Lower panes ratio of the NLO and corresponding LO distribution. The shaded band results from scale variations between $m_{t} / 2$ and $2 m_{t}$

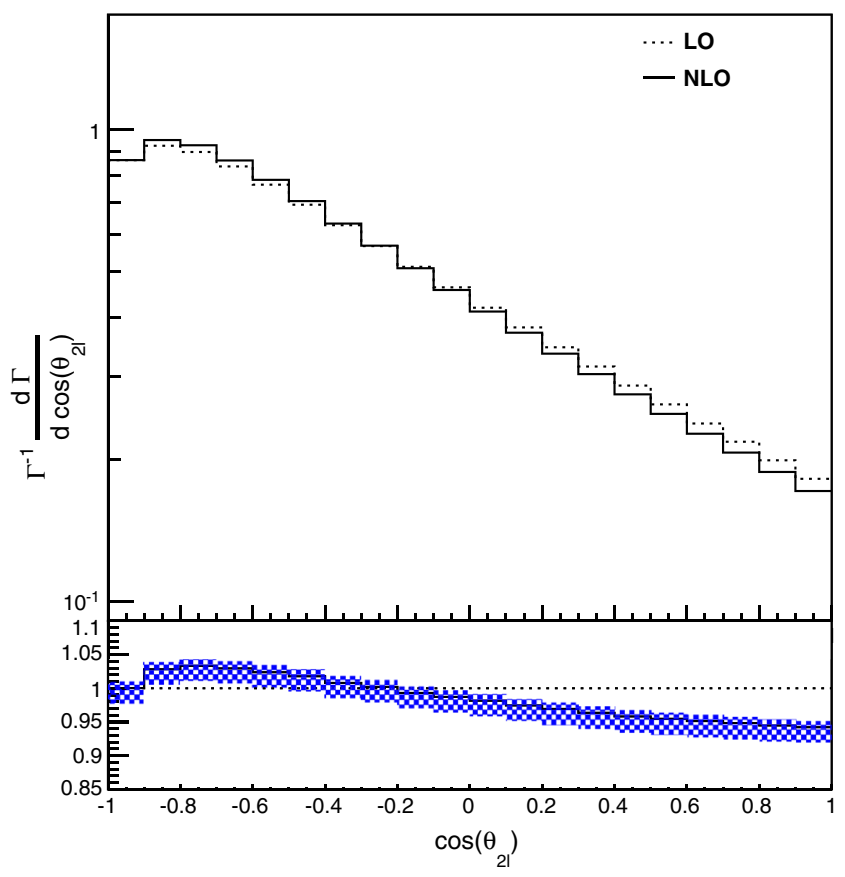

One may compare these $t$-spin analyzing powers with the corresponding ones of the dominant semileptonic decay modes $t \rightarrow b \ell^{+} \nu_{\ell}$. In the latter case one has $\kappa_{\ell}^{\mathrm{NLO}}=0.999$ [18] and $\kappa_{b}^{\mathrm{NLO}}=-0.39$ [19]. Moreover, in this inclusive case, $\kappa_{b}^{\mathrm{NLO}}=-\kappa_{W}^{\mathrm{NLO}}$. The charged lepton is the best top- 


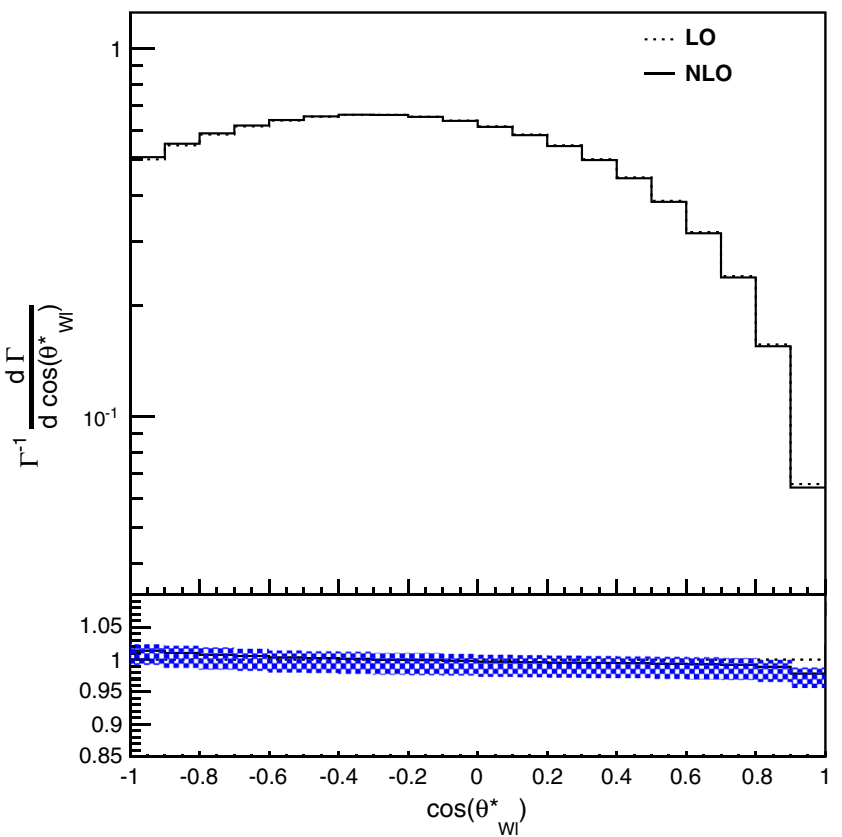

Fig. 10 Upper panes normalized distribution of $\cos \theta_{W l}^{*}$, where $\theta_{W l}^{*}$ is the angle between the $W^{+}$direction in the t rest frame and the lepton direction in the $W^{+}$rest frame (left). The right plot shows the normalized distribution of $\cos \theta_{W b}$, where $\theta_{W b}$ is the angle between the $W$ and

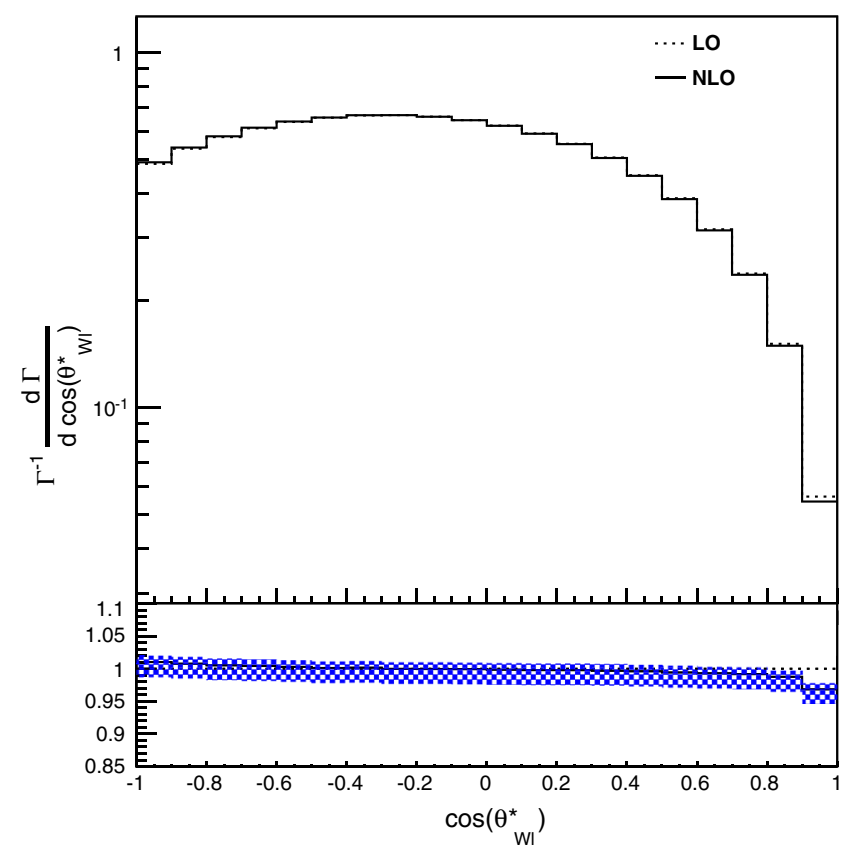

Fig. 11 Same as Fig. 10, but for $Y=0.001$

spin analyzer in the semileptonic decays both without and with an additional jet. This is due to the V-A structure of the charged weak current and angular momentum conservation. If an additional jet is produced in top-quark decay,

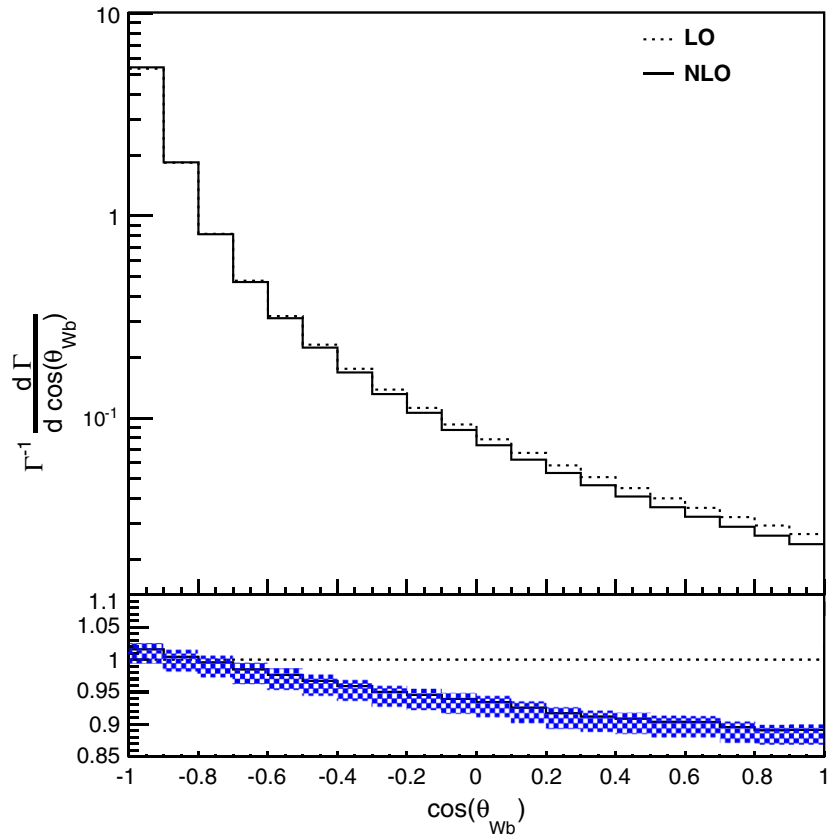

the b-jet directions in the $t$ rest frame. The jet resolution parameter is $Y=0.01$ and $\mu=m_{t}$. Lower panes ratio of the NLO and corresponding $\mathrm{LO}$ distribution. The shaded band results from scale variations between $m_{t} / 2$ and $2 m_{t}$

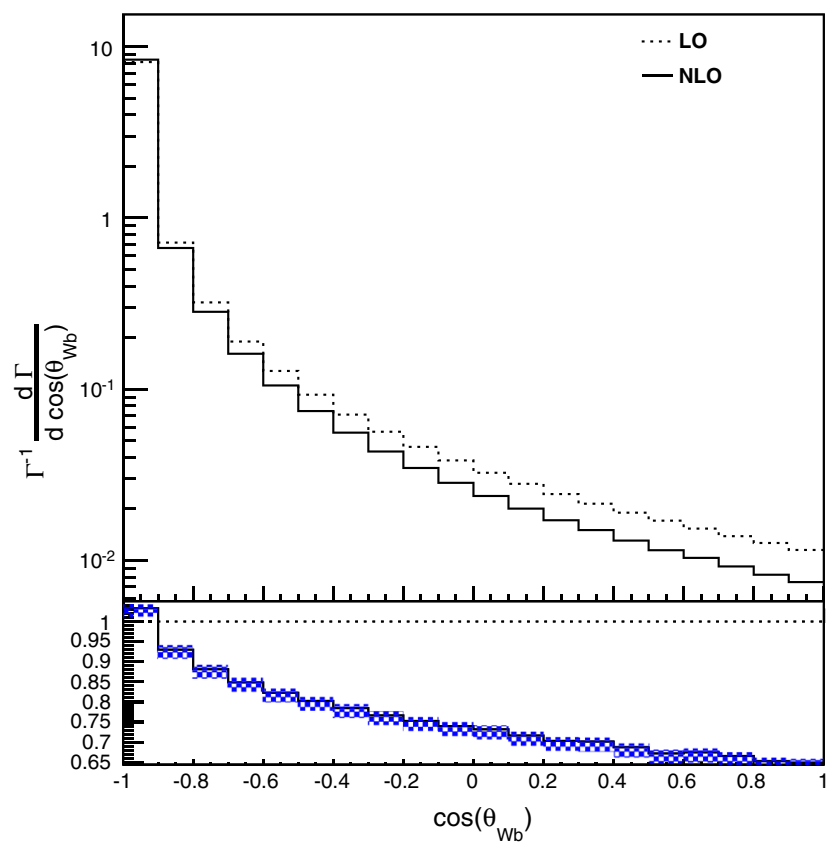

$\kappa_{b}=-\kappa_{W}$ no longer holds, of course, cf. Table 1. In semileptonic $t$ decays both without and with an additional jet the $t$-spin analyzing power of the $W$ boson is weaker than that of its daughter lepton $\ell^{+}$. This is due to the known fact that 
Table 1 Top-spin analyzing powers extracted from the normalized distributions (7) for $\mu=m_{t}$. The uncertainties due to scale variations between $m_{t} / 2$ and $2 m_{t}$ are below $1 \%$

\begin{tabular}{lcc}
\hline & $Y=0.01$ & $Y=0.001$ \\
\hline$\kappa_{\ell}^{\mathrm{LO}}$ & 0.981 & 0.993 \\
$\kappa_{\ell}^{\mathrm{NLO}}$ & 0.983 & 0.996 \\
$\kappa_{W}^{\mathrm{LO}}$ & 0.359 & 0.387 \\
$\kappa_{W}^{\mathrm{NLO}}$ & 0.351 & 0.381 \\
$\kappa_{b}^{\mathrm{LO}}$ & -0.326 & -0.368 \\
$\kappa_{b}^{\mathrm{NLO}}$ & -0.319 & -0.364 \\
\hline
\end{tabular}

for $t \rightarrow \ell^{+} \nu_{\ell} b(+\mathrm{jet})$, the amplitudes that correspond to the different polarization states of the intermediate $W$ boson interfere constructively (destructively) when $\ell^{+}$is emitted in (opposite to) the direction of the top spin.

\section{Summary}

We have computed the differential and total rate of the semileptonic decay of polarized top quarks $t \rightarrow \ell v_{\ell}+b$ jet + jet at next-to-leading order QCD. We have defined the jets by the Durham algorithm, and we have presented a number of distributions for two different values of the jet resolution parameter. The QCD corrections to the leading-order distributions are $\lesssim 5 \%$ in most of the kinematic range. Near kinematic edges or significantly off the $W$ resonance, the corrections can become $\sim 10 \%$. Our results should be useful as a building block for future analyses of top-quark production and decay in hadron and in $e^{+} e^{-}$collisions.

Acknowledgement The work of W.B. was supported by BMBF and that of C.M. by Deutsche Forschungsgemeinschaft through Graduiertenkolleg GRK 1675.

Open Access This article is distributed under the terms of the Creative Commons Attribution License which permits any use, distribution, and reproduction in any medium, provided the original author(s) and the source are credited.

Funded by $\mathrm{SCOAP}^{3}$ / License Version CC BY 4.0.

\section{Appendix}

In this appendix we collect, for convenience of the reader, the un-integrated and integrated subtraction terms that we used to handle the soft and collinear divergences which appear in the phase space integrals of the real radiation matrix elements of the processes (3) and in the 1-loop corrections to (2). We use the dipole subtraction method [34] and its extension to the decay of a massive quark, worked out in [33,36,37].

In this framework, the decay rate of (1) is given, as a function of the jet resolution parameter $Y$, at NLO QCD by $\Gamma^{\mathrm{NLO}}(Y)=\Gamma^{\mathrm{B}}(Y)+\delta \Gamma(Y)$, where $\delta \Gamma(Y)$

$$
\begin{aligned}
= & \int \mathrm{d} \phi_{4}\left(\delta \mathcal{M}_{V}^{(d)} F_{4}(Y)+F_{4}(Y) \int \mathrm{d} \phi^{(\text {dip. })} \delta \mathcal{M}_{\mathrm{CT}}^{(d)}\right)_{d=4} \\
& +\int \mathrm{d} \phi_{5}\left(\left(\mathcal{M}_{R}^{*} \mathcal{M}_{R}\right)^{(4)} F_{5}(Y)-\tilde{F}_{4}(Y) \delta \mathcal{M}_{\mathrm{CT}}^{(4)}\right) \cdot \quad(10)
\end{aligned}
$$

Here, $\mathrm{d} \phi_{4}, \mathrm{~d} \phi_{5}$ and $\mathrm{d} \phi^{(\mathrm{dip})}$ are the 4-particle, 5-particle, and dipole phase-space measures, respectively, $\delta \mathcal{M}_{\mathrm{CT}}$ denotes, schematically, the dipole subtraction counterterms for the two real radiation processes (3), and

$$
\begin{aligned}
& F_{4}(Y)=\Theta\left(Y_{b, g}-Y\right), \quad \tilde{F}_{4}(Y)=\Theta\left(\tilde{Y}_{(i j), l}-Y\right), \\
& F_{5}(Y)=\sum_{i, j \neq l} \Theta\left(Y_{i, j}-Y\right) \Theta\left(Y-Y_{(i j), l}\right)
\end{aligned}
$$

denote jet functions. The quantity $Y_{(i j), l}$ is calculated from the momentum of the pseudo-jet that consists of partons $i$ and $j$, cf. Sect. 2, whereas the quantity $\tilde{Y}_{(i j), l}$ is calculated from the emitter and spectator momenta $\tilde{k}_{i j}$ and $\tilde{k}_{l}$, which are defined in terms of the 5-particle phase space $\phi_{5}$. The following formulas are given for conventional dimensional regularization.

\section{Un-integrated dipoles}

The set of counterterms $\delta \mathcal{M}_{\mathrm{CT}}$ for the real radiation processes (3) can be constructed, using the emitter-spectator terminology of [34], with so-called final-final and final-initial dipoles. We denote the 4-momenta of the top quark and of the $b$ quark from the $t W b$ vertex with $k_{t}$ and $k_{b}$, and those of the two gluons or the $q, \bar{q}$ in (3) by $k_{1}, k_{2}$.

The final-final dipoles required for (3) can be obtained from [34]:

$$
\begin{aligned}
& \mathcal{D}_{b \rightarrow b g_{1}, g_{2}}^{\lambda_{1} \lambda_{2}}=\frac{-1}{2 k_{1} \cdot k_{b}} 4 \pi \alpha_{s} C_{F} \mu^{2 \epsilon} \\
& \quad \times\left[\delta^{\lambda_{1} \lambda_{2}}\left(\frac{2}{1-Z_{b 1,2}\left(1-Y_{b 1,2}\right)}-1-Z_{b 1,2}-\epsilon\left(1-Z_{b 1,2}\right)\right)\right], \\
& \mathcal{D}_{b \rightarrow b g_{2}, g_{1}}^{\lambda_{1} \lambda_{2}}=\frac{-1}{2 k_{2} \cdot k_{b}} 4 \pi \alpha_{s} C_{F} \mu^{2 \epsilon} \\
& \quad \times\left[\delta^{\lambda_{1} \lambda_{2}}\left(\frac{2}{1-Z_{b 2,1}\left(1-Y_{b 2,1}\right)}-1-Z_{b 2,1}-\epsilon\left(1-Z_{b 2,1}\right)\right)\right], \\
& \mathcal{D}_{g \rightarrow g g, b}^{\rho_{1} \rho_{2}}=\frac{-1}{2 k_{1} \cdot k_{2}} 8 \pi \alpha_{s} C_{A} \mu^{2 \epsilon} \\
& \quad \times\left[-g^{\rho_{1} \rho_{2}}\left(\frac{1}{1-Z_{12, b}\left(1-Y_{12, b}\right)}+\frac{1}{1-\left(1-Z_{12, b}\right)\left(1-Y_{12, b}\right)}-2\right)\right. \\
& \left.\quad+\frac{1-\epsilon}{k_{1} \cdot k_{2}} \Pi_{\mathrm{FF}}^{\rho_{1}} \Pi_{\mathrm{FF}}^{\rho_{2}}\right], \\
& \mathcal{D}_{g \rightarrow q \bar{q}, b}^{\rho_{1} \rho_{2}}=\frac{-1}{2 k_{1} \cdot k_{2}} 4 \pi \alpha_{s} N_{f} \mu^{2 \epsilon}\left[-g^{\rho_{1} \rho_{2}}-\frac{2}{k_{1} \cdot k_{2}} \Pi_{\mathrm{FF}}^{\rho_{1}} \Pi_{\mathrm{FF}}^{\rho_{2}}\right] .
\end{aligned}
$$

Here $\epsilon=(4-d) / 2, C_{F}=\left(N_{c}^{2}-1\right) /\left(2 N_{c}\right), C_{A}=N_{c}$, $N_{f}=5$, and 
$Y_{i j, l}=\frac{k_{i} \cdot k_{j}}{k_{i} \cdot k_{j}+\left(k_{i}+k_{j}\right) \cdot k_{l}}$,

$Z_{i j, l}=\frac{k_{i} \cdot k_{l}}{\left(k_{i}+k_{j}\right) \cdot k_{l}},(l \neq i, j)$,

$\Pi_{\mathrm{FF}}^{\rho_{1}}=\left(1-Z_{12, b}\right) k_{1}^{\rho_{1}}-Z_{12, b} k_{2}^{\rho_{1}}$.

The indices $\lambda_{i}$ and $\rho_{i}$ transform according to the spinor and vector representations of the Lorentz group, i.e., they refer to the spin of the initial-state quark and gluon, respectively, in (11).

The final-initial dipoles for $t \rightarrow W b g_{1} g_{2}$ were constructed in [33,36] (using, in part, results of [37]). They contain the eikonal terms $\propto m_{t}^{2} k_{i} \cdot k_{j} /\left(k_{t} \cdot k_{i}\right)^{2}$ for canceling the soft singularities that arise from gluon radiation off the initial top quark. The final-initial dipole for $t \rightarrow W b q \bar{q}$ can be constructed analogously.

$$
\begin{aligned}
& \mathcal{D}_{b \rightarrow b g_{1}}^{t \lambda_{1} \lambda_{2}}=\frac{-1}{2 k_{1} \cdot k_{b}} 4 \pi \alpha_{s} C_{F} \mu^{2 \epsilon} \\
& \times\left[\delta^{\lambda_{1} \lambda_{2}}\left(\frac{2}{1-Z_{b 1}^{t}}-1-Z_{b 1}^{t}-\epsilon Y_{b 1}^{t}\left(1-Z_{b 1}^{t}\right)-\frac{m_{t}^{2} k_{1} \cdot k_{b}}{\left(k_{t} \cdot k_{1}\right)^{2}}\right)\right], \\
& \mathcal{D}_{b \rightarrow b g_{2}}^{t \lambda_{1} \lambda_{2}}=\frac{-1}{2 k_{2} \cdot k_{b}} 4 \pi \alpha_{s} C_{F} \mu^{2 \epsilon} \\
& \times\left[\delta^{\lambda_{1} \lambda_{2}}\left(\frac{2}{1-Z_{b 2}^{t}}-1-Z_{b 2}^{t}-\epsilon Y_{b 2}^{t}\left(1-Z_{b 2}^{t}\right)-\frac{m_{t}^{2} k_{2} \cdot k_{b}}{\left(k_{t} \cdot k_{2}\right)^{2}}\right)\right], \\
& \mathcal{D}_{g \rightarrow g g}^{t \rho_{1} \rho_{2}}=\frac{-1}{2 k_{1} \cdot k_{2}} 8 \pi \alpha_{s} C_{A} \mu^{2 \epsilon} \\
& \quad \times\left[-g^{\rho_{1} \rho_{2}}\left(\frac{1-Z_{12}^{t}}{Z_{12}^{t}}+\frac{1-Z_{21}^{t}}{Z_{21}^{t}}-\frac{m_{t}^{2} k_{1} \cdot k_{2}}{2\left(k_{t} \cdot k_{1}\right)^{2}}-\frac{m_{t}^{2} k_{1} \cdot k_{2}}{2\left(k_{t} \cdot k_{2}\right)^{2}}\right)\right. \\
& \left.\quad+\frac{1-\epsilon}{k_{1} \cdot k_{2}} \Pi_{\mathrm{FI}}^{\rho_{1}} \Pi_{\mathrm{FI}}^{\rho_{2}}\right], \\
& \mathcal{D}_{g \rightarrow q \bar{q}}^{t \rho_{1} \rho_{2}}=\frac{-1}{2 k_{1} \cdot k_{2}} 4 \pi \alpha_{s} N_{f} \mu^{2 \epsilon}\left[-g^{\rho_{1} \rho_{2}}-\frac{2}{k_{1} \cdot k_{2}} \Pi_{\mathrm{FI}}^{\rho_{1}} \Pi_{\mathrm{FI}}^{\rho_{2}}\right] .
\end{aligned}
$$

Here

$Z_{i j}^{t}=\frac{2 k_{t} \cdot k_{i}}{m_{t}^{2}\left(1-r_{i j}^{2}\right)}, \quad Y_{i j}^{t}=\frac{2 k_{i} \cdot k_{j}}{m_{t}^{2}\left(1-r_{i j}\right)^{2}}$,

$r_{i j}=\frac{\left(k_{t}-k_{i}-k_{j}\right)^{2}}{m_{t}^{2}}$.

In this case the vector $\Pi_{\mathrm{FI}}^{\rho}$ takes a more complicated form. For the sake of brevity we refer to Eq. (20) of [33].

\section{Integrated dipoles}

For the analytical integration of the final-final dipoles over the respective subspaces we use a phase-space splitting of the form

$\mathrm{d} \phi_{5}\left(k_{i}, k_{j}, k_{l}, \ldots\right)=\mathrm{d} \phi_{4}\left(\tilde{k}_{i j}, \tilde{k}_{l}, \ldots\right) \times \mathrm{d} \phi_{i j, l}^{(\mathrm{dip} .)}\left(Y_{i j, l}, Z_{i j, l}\right)$,

such that one can, in the end, identify $\mathrm{d} \phi_{4}\left(\tilde{k}_{i j}, \tilde{k}_{l}, \ldots\right)$ with the four-particle phase space of the Born matrix elements or of the virtual corrections.
The momenta of the emitter $\tilde{k}_{i j}$ and the spectator $\tilde{k}_{l}$ are constructed according to [34] from the soft/collinear pair $k_{i}$, $k_{j}$ and another parton momentum $k_{l}$, whereas all remaining momenta are unaffected. In our case the emitter and spectator is either a b-quark or a gluon, i.e. $\tilde{k}_{i j}=\tilde{k}_{b / g}$ and $\tilde{k}_{l}=\tilde{k}_{g / b}$.

The phase space of the final-final dipoles can then be parameterized as

$$
\begin{aligned}
& \mathrm{d} \phi_{i j, l}^{(\mathrm{dip} .)}\left(Y_{i j, l}, Z_{i j, l}\right)=\left[\frac{\left(2 \tilde{k}_{b} \cdot \tilde{k}_{g}\right)^{1-\epsilon}\left[\mathrm{d} \Omega_{(d-3)}\right]}{16 \pi^{2}(2 \pi)^{1-2 \epsilon}}\right. \\
& \left.\times \frac{\Theta\left(Z_{i j, l}\left(1-Z_{i j, l}\right)\right) \mathrm{d} Z_{i j, l}}{\left(Z_{i j, l}\left(1-Z_{i j, l}\right)\right)^{\epsilon}} \frac{\Theta\left(Y_{i j, l}\left(1-Y_{i j, l}\right)\right) \mathrm{d} Y_{i j, l}}{\left(1-Y_{i j, l}\right)^{2 \epsilon-1} Y_{i j, l}^{\epsilon}}\right] .
\end{aligned}
$$

The double index $i j$ labels the soft/collinear pair and the index $l$ refers to the momentum of the remaining final-state parton.

Integration of (11) over the dipole phase space yields

$$
\begin{aligned}
& D_{b \rightarrow b g_{1}, g_{2}}^{\lambda_{1} \lambda_{2}}=\frac{\alpha_{s}}{4 \pi} \frac{-C_{F}}{\Gamma(1-\epsilon)}\left(\frac{\tilde{\mu}^{2}}{\tilde{S}_{b g}}\right)^{\epsilon} \\
& \times\left[\delta^{\lambda_{1} \lambda_{2}}\left(\frac{1}{\epsilon^{2}}+\frac{3}{2 \epsilon}+5-\frac{\pi^{2}}{2}+\mathcal{O}(\epsilon)\right)\right], \\
& D_{b \rightarrow b g_{2}, g_{1}}^{\lambda_{1} \lambda_{2}}=\frac{\alpha_{s}}{4 \pi} \frac{-C_{F}}{\Gamma(1-\epsilon)}\left(\frac{\tilde{\mu}^{2}}{\tilde{S}_{b g}}\right)^{\epsilon} \\
& \quad \times\left[\delta^{\lambda_{1} \lambda_{2}}\left(\frac{1}{\epsilon^{2}}+\frac{3}{2 \epsilon}+5-\frac{\pi^{2}}{2}+\mathcal{O}(\epsilon)\right)\right], \\
& D_{g \rightarrow g g, b}^{\rho_{1} \rho_{2}}=\frac{\alpha_{s}}{4 \pi} \frac{-2 C_{A}}{\Gamma(1-\epsilon)}\left(\frac{\tilde{\mu}^{2}}{\tilde{S}_{b g}}\right)^{\epsilon} \\
& \quad \times\left[-g^{\rho_{1} \rho_{2}}\left(\frac{1}{\epsilon^{2}}+\frac{11}{6 \epsilon}+\frac{50}{9}-\frac{\pi^{2}}{2}+\mathcal{O}(\epsilon)\right)\right], \\
& D_{g \rightarrow q \bar{q}, b}^{\rho_{1} \rho_{2}}=\frac{\alpha_{s}}{4 \pi} \frac{-N_{f}}{\Gamma(1-\epsilon)}\left(\frac{\tilde{\mu}^{2}}{\tilde{S}_{b g}}\right)^{\epsilon} \\
& \quad \times\left[-g^{\rho_{1} \rho_{2}}\left(-\frac{2}{3 \epsilon}-\frac{16}{9}+\mathcal{O}(\epsilon)\right)\right] .
\end{aligned}
$$

Here $\tilde{\mu}^{2}=4 \pi \mu^{2}$ and $\tilde{S}_{b g}=\left(\tilde{k}_{b}+\tilde{k}_{g}\right)^{2} / m_{t}^{2}=2 \tilde{k}_{b} \cdot \tilde{k}_{g} / m_{t}^{2}$.

In the case of the final-initial dipoles the phase-space splitting takes a slightly different form:

$\mathrm{d} \phi_{5}\left(k_{i}, k_{j}, R\right)=\mathrm{d} \phi_{4}\left(\tilde{k}_{i j}, \tilde{R}\right) \times \mathrm{d} \phi_{i j}^{t(\text { dip. })}\left(Y_{i j}^{t}, Z_{i j}^{t}\right)$.

Again $\mathrm{d} \phi_{4}\left(\tilde{k}_{i j}, \tilde{R}\right)$ can be identified with the phase space of the Born matrix elements or of the virtual corrections. Here, the phase-space mapping $5 \rightarrow 4$ affects, besides the soft/collinear pair, also all other final-state momenta, denoted by $R$. 
This procedure, as well as the dipole phase-space parameterization,

$$
\begin{gathered}
\mathrm{d} \phi_{i j}^{(\text {dip. })}\left(Y_{i j}^{t}, Z_{i j}^{t}\right)=\left[\frac{\left(m_{t}^{2}\right)^{1-\epsilon}\left[\mathrm{d} \Omega_{(d-3)}\right]}{16 \pi^{2}(2 \pi)^{1-2 \epsilon}}\left(1-r_{i j}\right)^{2}\left(\frac{1+r_{i j}}{1-r_{i j}}\right)^{2 \epsilon}\right. \\
\left.\times \frac{\Theta\left(Z_{i j}^{t}\left(1-Z_{i j}^{t}\right)\right) \mathrm{d} Z_{i j}^{t}}{\left(Z_{i j}^{t}+r_{i j}^{2}\left(1-Z_{i j}^{t}\right)\right)^{\epsilon}} \frac{\Theta\left(Y_{i j}^{t}\left(Y_{\max }-Y_{i j}^{t}\right)\right) \mathrm{d} Y_{i j}^{t}}{\left(Y_{\max }-Y_{i j}^{t}\right)^{\epsilon}\left(Y_{i j}^{t}\right)^{\epsilon}}\right],
\end{gathered}
$$

is adapted from [33,37].

The boundary of the $Y_{i j}^{t}$ integration is

$$
Y_{\max }=\frac{\left(1+r_{i j}\right)^{2} Z_{i j}^{t}\left(1-Z_{i j}^{t}\right)}{\left(1-Z_{i j}^{t}\right)+r_{i j}^{2} Z_{i j}^{t}} .
$$

Integration of (12) over the dipole phase space yields hypergeometric functions ${ }_{2} \mathrm{~F}_{1}$, which we expanded in powers of $\epsilon$ using the package HPL 2.0 [45]. We obtain

$$
\begin{aligned}
& D_{b \rightarrow b g_{1}}^{t \lambda_{1} \lambda_{2}}=\frac{\alpha_{s}}{4 \pi} \frac{-C_{F}}{\Gamma(1-\epsilon)} \tilde{\mu}^{2 \epsilon} \\
& \quad \times\left[\delta^{\lambda_{1} \lambda_{2}}\left(\frac{1}{\epsilon^{2}}+\frac{5-4 \ln \left(1-\tilde{T}_{b}\right)}{2 \epsilon}+F_{b g}+\mathcal{O}(\epsilon)\right)\right] \\
& D_{b \rightarrow b g_{2}}^{t \lambda_{1} \lambda_{2}}=\frac{\alpha_{s}}{4 \pi} \frac{-C_{F}}{\Gamma(1-\epsilon)} \tilde{\mu}^{2 \epsilon} \\
& \quad \times\left[\delta^{\lambda_{1} \lambda_{2}}\left(\frac{1}{\epsilon^{2}}+\frac{5-4 \ln \left(1-\tilde{T}_{b}\right)}{2 \epsilon}+F_{b g}+\mathcal{O}(\epsilon)\right)\right], \\
& D_{g \rightarrow g g}^{t \rho_{1} \rho_{2}}=\frac{\alpha_{s}}{4 \pi} \frac{-2 C_{A}}{\Gamma(1-\epsilon)} \tilde{\mu}^{2 \epsilon} \\
& \quad \times\left[-g^{\rho_{1} \rho_{2}}\left(\frac{1}{\epsilon^{2}}+\frac{17-12 \ln \left(1-\tilde{T}_{g}\right)}{6 \epsilon}+F_{g g}+\mathcal{O}(\epsilon)\right)\right], \\
& D_{g \rightarrow q \bar{q}}^{t \rho_{1} \rho_{2}}=\frac{\alpha_{s}}{4 \pi} \frac{-N_{f}}{\Gamma(1-\epsilon)} \tilde{\mu}^{2 \epsilon} \\
& \quad \times\left[-g^{\rho_{1} \rho_{2}}\left(-\frac{2}{3 \epsilon}+F_{q \bar{q}}+\mathcal{O}(\epsilon)\right)\right],
\end{aligned}
$$

where

$$
\begin{gathered}
F_{b g}=-\ln \left(\tilde{T}_{b}\right) \frac{\tilde{T}_{b}\left(6-7 \tilde{T}_{b}\right)}{2\left(1-\tilde{T}_{b}\right)^{2}}+\frac{27-25 \tilde{T}_{b}}{4\left(1-\tilde{T}_{b}\right)} \\
+2 \operatorname{Li}_{2}\left(1-\tilde{T}_{b}\right)-\frac{5}{6} \pi^{2}-5 \ln \left(1-\tilde{T}_{b}\right)+2 \ln ^{2}\left(1-\tilde{T}_{b}\right), \\
F_{g g}=-\ln \left(\tilde{T}_{g}\right) \frac{\tilde{T}_{g}\left(24-84 \tilde{T}_{g}+134 \tilde{T}_{g}^{2}-91 \tilde{T}_{g}^{3}+23 \tilde{T}_{g}^{4}\right)}{6\left(1-\tilde{T}_{g}\right)^{5}} \\
+\frac{901-3694 \tilde{T}_{g}+5326 \tilde{T}_{g}^{2}-3534 \tilde{T}_{g}^{3}+881 \tilde{T}_{g}^{4}}{120\left(1-\tilde{T}_{g}\right)^{4}} \\
\quad+2 \operatorname{Li}_{2}\left(1-\tilde{T}_{g}\right)-\frac{5}{6} \pi^{2}-\frac{17}{3} \ln \left(1-\tilde{T}_{g}\right)+2 \ln ^{2}\left(1-\tilde{T}_{g}\right),
\end{gathered}
$$

$$
\begin{gathered}
F_{q \bar{q}}=\ln \left(\tilde{T}_{g}\right) \frac{\tilde{T}_{g}\left(3+8 \tilde{T}_{g}^{2}-7 \tilde{T}_{g}^{3}+2 \tilde{T}_{g}^{4}\right)}{3\left(1-\tilde{T}_{g}\right)^{5}}+\frac{4}{3} \ln \left(1-\tilde{T}_{g}\right) \\
-\frac{\left(101-494 \tilde{T}_{g}+526 \tilde{T}_{g}^{2}-334 \tilde{T}_{g}^{3}+81 \tilde{T}_{g}^{4}\right)}{60\left(1-\tilde{T}_{g}\right)^{4}} .
\end{gathered}
$$

Here $\tilde{T}_{b}=\left(k_{t}-\tilde{k}_{b}\right)^{2} / m_{t}^{2}$, and $\tilde{T}_{g}=\left(k_{t}-\tilde{k}_{g}\right)^{2} / m_{t}^{2}$. Equation (15) agrees with the result of [36], and Eqs. (16) and (17) with those of [33].

\section{References}

1. F.-P. Schilling, Int. J. Mod. Phys. A 27, 1230016 (2012). [arXiv: 1206.4484 [hep-ex]]

2. F. Deliot et al., CDF and D0 Collaborations. Int. J. Mod. Phys. A 28, 1330013 (2013). [arXiv:1302.3628 [hep-ex]]

3. E. Yazgan, arXiv:1308.3338 [hep-ex]

4. R. Chierici, Phys. Scripta T 158, 014007 (2013)

5. P. Baernreuther, M. Czakon, A. Mitov, Phys. Rev. Lett. 109, 132001 (2012). [arXiv:1204.5201 [hep-ph]]

6. M. Czakon, P. Fiedler, A. Mitov, Phys. Rev. Lett. 110, 252004 (2013). [arXiv:1303.6254 [hep-ph]]

7. J. Gao, C.S. Li, H.X. Zhu, Phys. Rev. Lett. 110, 042001 (2013). [arXiv:1210.2808 [hep-ph]]

8. M. Brucherseifer, F. Caola, K. Melnikov, JHEP 1304, 059 (2013). [arXiv:1301.7133 [hep-ph]]

9. M. Jezabek, J.H. Kühn, Nucl. Phys. B 314, 1 (1989)

10. M. Jezabek, J.H. Kühn, Phys. Rev. D 48, 1910 (1993) [Erratumibid. D 49, 4970 (1994)] [arXiv:hep-ph/9302295]

11. A. Denner, T. Sack, Nucl. Phys. B 358, 46 (1991)

12. G. Eilam, R.R. Mendel, R. Migneron, A. Soni, Phys. Rev. Lett. 66, 3105 (1991)

13. A. Czarnecki, K. Melnikov, Nucl. Phys. B 544, 520 (1999). [arXiv: hep-ph/9806244]

14. K.G. Chetyrkin, R. Harlander, T. Seidensticker, M. Steinhauser, Phys. Rev. D 60, 114015 (1999). [arXiv:hep-ph/9906273]

15. A. Czarnecki, J.G. Körner, J.H. Piclum, Phys. Rev. D 81, 111503 (2010). [arXiv:1005.2625 [hep-ph]]

16. H.S. Do, S. Groote, J.G. Körner, M.C. Mauser, Phys. Rev. D 67, 091501 (2003). [hep-ph/0209185]

17. M. Jezabek, J.H. Kühn, Nucl. Phys. B 320, 20 (1989)

18. A. Czarnecki, M. Jezabek, J.H. Kühn, Nucl. Phys. B 351, 70 (1991)

19. A. Brandenburg, Z.G. Si, P. Uwer, Phys. Lett. B 539, 235 (2002). [hep-ph/0205023]

20. M. Fischer, S. Groote, J.G. Körner, M.C. Mauser, Phys. Rev. D 65, 054036 (2002). [arXiv:hep-ph/0101322]

21. S. Groote, W.S. Huo, A. Kadeer, J.G. Körner, Phys. Rev. D 76, 014012 (2007). [hep-ph/0602026]

22. K. Hagiwara, K. Mawatari, H. Yokoya, JHEP 0712, 041 (2007). [arXiv:0707.3194 [hep-ph]]

23. A. Kadeer, J.G. Körner, arXiv:0906.3474 [hep-ph]

24. A. Ali, E.A. Kuraev, Y.M. Bystritskiy, Eur. Phys. J. C 67, 377 (2010). [arXiv:0911.3027 [hep-ph]]

25. Y. Kitadono, H.-n. Li, Phys. Rev. D 87, 054017 (2013). [arXiv: 1211.3260 [hep-ph]]

26. G. Corcella, A.D. Mitov, Nucl. Phys. B 623, 247 (2002). [hep-ph/ 0110319]

27. M. Cacciari, G. Corcella, A.D. Mitov, JHEP 0212, 015 (2002). [hep-ph/0209204] 
28. G. Corcella, F. Mescia, Eur. Phys. J. C 65, 171 (2010). [Erratumibid. C 68, 687 (2010)] [arXiv:0907.5158 [hep-ph]]

29. B.A. Kniehl, G. Kramer, S.M.M. Nejad. Nucl. Phys. B 862, 720 (2012). [arXiv:1205.2528 [hep-ph]]

30. S. M. M. Nejad, arXiv:1310.5686 [hep-ph]

31. S.M.M. Nejad, arXiv:1310.5686 [hep-ph]

32. S. Dittmaier, P. Uwer, S. Weinzierl, Eur. Phys. J. C 59, 625 (2009). [arXiv:0810.0452 [hep-ph]]

33. K. Melnikov, A. Scharf, M. Schulze, Phys. Rev. D 85, 054002 (2012). [arXiv:1111.4991 [hep-ph]]

34. S. Catani, M.H. Seymour, Nucl. Phys. B 485, 291 (1997) [Erratumibid. B 510,503 (1998)] [hep-ph/9605323]

35. S. Catani, S. Dittmaier, M.H. Seymour, Z. Trocsanyi, Nucl. Phys. B 627, 189 (2002). [hep-ph/0201036]

36. K. Melnikov, M. Schulze, A. Scharf, Phys. Rev. D 83, 074013 (2011). [arXiv:1102.1967 [hep-ph]]

37. J.M. Campbell, R.K. Ellis, F. Tramontano, Phys. Rev. D 70, 094012 (2004). [hep-ph/0408158]
38. G. Passarino, M.J.G. Veltman, Nucl. Phys. B 160, 151 (1979)

39. R.K. Ellis, G. Zanderighi, JHEP 0802, 002 (2008). [arXiv:0712. 1851 [hep-ph]]

40. S. Catani, Y.L. Dokshitzer, M. Olsson, G. Turnock, B.R. Webber, Phys. Lett. B 269, 432 (1991)

41. K. Melnikov, M. Schulze, JHEP 0908, 049 (2009). [arXiv:0907. 3090 [hep-ph]]

42. A. Denner, S. Dittmaier, S. Kallweit, S. Pozzorini, JHEP 1210, 110 (2012) . [arXiv:1207.5018 [hep-ph]]

43. F. Cascioli, S. Kallweit, P. Maierhfer, S. Pozzorini, arXiv:1312. 0546 [hep-ph]

44. K. Kroeninger, in Talk Given at 6th International Workshop on Top-Quark Physics, Durbach (Germany) 14-19 September 2013

45. D. Maitre, Comput. Phys. Commun. 174, 222 (2006). [hep-ph/ 0507152] 